\title{
Aporte de biomasa aérea de las especies arbóreas de la familia Myristicaceae en los bosques Amazónicos del Perú
}

\author{
Marisabel Ureta Adrianzén ${ }^{1,2}$
}

1. Jardín Botánico de Missouri, Programa-Perú. Prolg. Bolognesi Mz E-6, Oxapampa, Perú; marisabelureta@gmail.com

2. Máster Oficial en Biodiversidad en áreas tropicales y su conservación, Universidad Internacional Menéndez Pelayo (UIMP), c/Isaac Peral 23, Madrid, España

Recibido 29-IV-2014. Corregido 01-VIII-2014. Aceptado 09-IX-2014.

\begin{abstract}
Aboveground biomass input of Myristicaceae tree species in the Amazonian Forest in Perú. Amazonian forests are a vast storehouse of biodiversity and function as carbon sinks from biomass that accumulates in various tree species. In these forests, the taxa with the greatest contribution of biomass cannot be precisely defined, and the representative distribution of Myristicaceae in the Peruvian Amazon was the starting point for designing the present study, which aimed to quantify the biomass contribution of this family. For this, I analyzed the databases that corresponded to 38 sample units that were previously collected and that were provided by the TeamNetwork and RAINFOR organizations. The analysis consisted in the estimation of biomass using pre-established allometric equations, Kruskal-Wallis sample comparisons, interpolation-analysis maps, and nonparametric multidimensional scaling (NMDS). The results showed that Myristicaceae is the fourth most important biomass contributor with $376.97 \mathrm{Mg} / \mathrm{ha}(9.92 \mathrm{Mg} / \mathrm{ha}$ in average), mainly due to its abundance. Additionally, the family shows a noticeable habitat preference for certain soil conditions in the physiographic units, such is the case of Virola pavonis in "varillales", within "floodplain", or Iryanthera tessmannii and Virola loretensis in sewage flooded areas or "igapó" specifically, and the preference of Virola elongata and Virola surinamensis for white water flooded areas or "várzea" edaphic conditions of the physiographic units taken in the study. Rev. Biol. Trop. 63 (1): 263-273. Epub 2015 March 01.
\end{abstract}

Key words: aboveground biomass, Peruvian Amazon, Myristicaceae, preferences, physiographic units.

Los bosques tropicales han captado un gran interés debido a que contribuyen en la captura de $\mathrm{CO}_{2}$ en función de la biomasa aérea proporcionada por innumerables especies vegetales (Grace, 2004), y alteraciones en ellos pueden influir fuertemente en las emisiones y la fijación de carbono en estos ecosistemas (Keller et al., 2004; Magnusson et al., 2008; Vieira et al., 2008). Dentro de ellos, los bosques amazónicos son los que presentan una especial atención en la conservación de la biodiversidad. Según Gentry (1986) los bosques amazónicos de Sudamérica son los más ricos en especies arbóreas, estimando alrededor de 90000 especies de plantas vasculares, y son considerados como el componente principal de los bosques húmedos tropicales (Gentry,
1982). Además, la curiosa duración de la estacionalidad en los bosques amazónicos tiene una correlación significativa con la distribución de la biomasa aérea (Saatchi, Houghton, Dos Santos, Soares, \& Yu, 2007), ocasionando efectos importantes sobre la variación geográfica en la composición florística de árboles (Freitas et al., 2005; Baker et al., 2009; Prentice et al., 2001) los cuales pueden llegar a acumular alrededor de $120 \pm 30 \mathrm{Pg}$ de carbono a partir de la biomasa aérea (Malhi et al., 2006), lo que demuestra que son uno de los reservorios de carbono más grandes del mundo (Baker et al., 2004a).

En términos neotropicales, Perú es uno de los países con más de la mitad de su superficie cubierta por bosques tropicales (Pennington Reynel, \& Daza, 2004), siendo los bosques 
amazónicos los más representativos por su exuberante riqueza de especies leñosas, como cualidad más relevante desde el punto de vista florístico (Gentry, \& Ortiz, 1993). En estos bosques, la familia Myristicaceae R. Br. es una de las 10 familias más diversas e importantes de árboles (Javonec, 2002; Pennington et al., 2004). Comprende aproximadamente entre 350 a 500 especies distribuidas entre 17 y 19 géneros (Gentry, 1982; Pascal, \& Pelissier, 1996; Poulsen, Nelson, Tan, \& Balsleb, 1996; Spichiger, Loizeau, Latou, \& Barriera, 1996), ubicados en zonas tropicales de África, Asia y con pocos representantes en Oceanía. En América, se han registrado principalmente en Centroamérica y la Cuenca Amazónica.

El presente estudio se dedica a la familia Myristicaceae R. Br., ya que en el Perú, en términos taxonómicos, sólo cuenta con un registro de 42 especies distribuidas en cinco géneros (Brako, \& Zarucchi, 1993, Ulloa-Ulloa, Zarucchi, \& León, 2004, Vásquez, Rojas, \& Van der Werff, 2010). Así mismo, en términos ecológicos, forma parte representativa en la composición florística y dinámica de los ecosistemas amazónicos (Javonec, 2002; Ribeiro et al., 1999). El objetivo fue evidenciar el grado de representatividad en la cuantificación de biomasa aérea tienen las Myristicaceae $\mathrm{R}$. Br. en los bosques amazónicos del Perú a partir de las ecuaciones alométricas de Chave et al. (2005), obtenidas en los bosques húmedos tropicales de Australia, Brasil, Comboya, Colombia, Costa Rica, Guyana Francesa, India, Jamaica, Indonesia, Malasia, México, Nueva Guinea, Puerto Rico y Venezuela, pero aún no estimadas en Perú.

\section{MATERIALES Y MÉTODOS}

Área de estudio: El área de estudio comprendió la superficie de los bosques amazónicos del Perú, para lo que se consideró unidades muestrales denominadas parcelas permanentes de muestreo (de ahora en adelante PPM), ubicadas en tres departamentos amazónicos (hasta $1000 \mathrm{msnm})$, y pertenecientes a la zona de vida de Bosques Húmedos Tropicales (bh-T), (Holdridge, 1947):

a. Loreto (98-146msnm, con 14 PPM): Unidades muestrales ubicadas en la provincia de Maynas, en las Reservas Allpahuayo Mishana, Explornapo Camp, Explorama Lodge y Jenaro Herrera (Vásquez, 1997).

b. Pasco (414-859msnm, con cinco PPM): Unidades muestrales se ubican dentro del Parque Nacional Yanachaga Chemillén (Vásquez, Rojas, Monteagudo, \& Meza, 2005).

c. Madre de Dios (190-225msnm, con 19 PPM): Unidades muestrales se encuentran dentro de la Reserva Nacional Tambopata y el Parque Nacional Manu (Cornejo, Janovec, \& Tobler, 2006). En general, las unidades muestrales están ubicadas en áreas de bosques de planicie inundable y bosques de tierra firme, principalmente (Fig. 1).

Selección de muestra: Consideramos como unidad muestral a cada parcela permanente de muestreo (PPM). Los datos de las parcelas fueron proporcionados por las organizaciones RAINFOR y TeamNetwork que realizaron muestreos desde 1975 al 2012. Cada PPM tuvo una medida de $100 \times 100 \mathrm{~m}$, con un área de 1 ha, subdividida en 25 subparcelas de 400 $\mathrm{m}^{2}$ (20x20m), (Phillips, \& Baker, 2003). Para este estudio se consideró 38PPM, con las medidas de $\mathrm{DAP} \geq 10 \mathrm{~cm}$ y registros de identificaciones taxonómicas a nivel de familia, género y especie, de cada árbol registrado.

Estimación de la biomasa aérea (AGB est): El aporte de biomasa aérea fue obtenido a partir de la ecuación alométrica (a) para bosques lluviosos (>3500mm/a) de Chave et al. (2005), en la cual se utilizan las medidas de DAP (D) (cm), y densidad de madera $(p)(\mathrm{g} /$ $\mathrm{cm}^{3}$ ); ésta última medida tomada por especie de las tablas de Chave et al., 2006a. La biomasa aérea fue determinada en megagramos/hectárea para mayor comodidad del proceso de los datos 

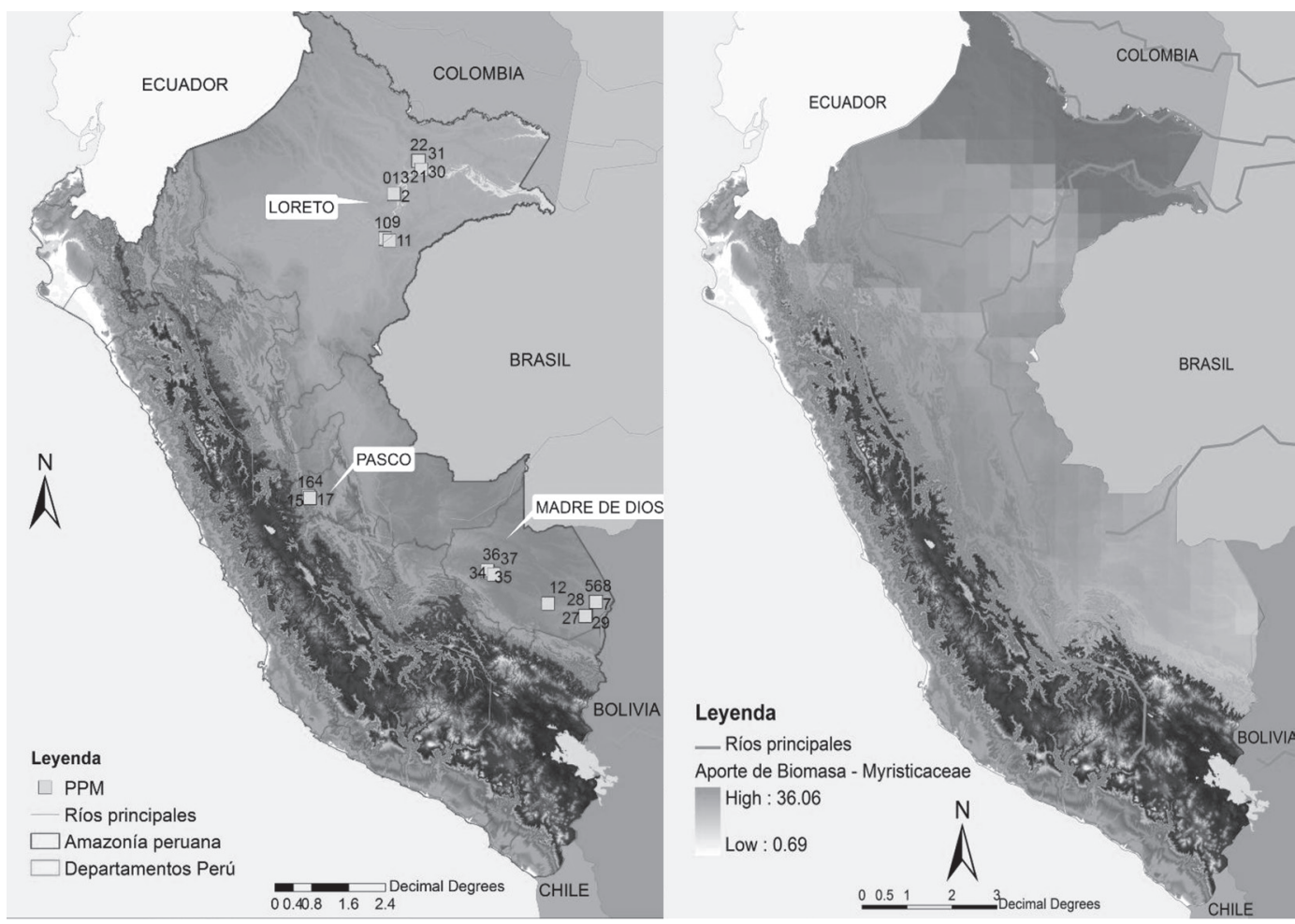

Fig. 1. Ubicación según ID y distribución de la biomasa aérea de las especies de Myristicaceae R. Br. obtenida en las 38 PPM.

Fig. 1. Location and distribution maps of aboveground biomass for Myristicaceae R. Br. species in the 38 PPM.

(a)

$(\mathrm{AGB})_{\text {est }}$ pxexp $\left(-1.239+1.980 \ln (\mathrm{D})+0.207(\ln (\mathrm{D}))^{2}-0.0281(\ln (\mathrm{D}))^{3}\right)$

Una vez obtenido este valor, se desarrollaron los mapas de distribución interpolando la acumulación de biomasa aérea de las especies de la familia Myristicaceae R. Br., utilizando como la máscara de superficie al área amazónica denominado también "Amazonía peruana", mediante el análisis de superficie GIS utilizando el programa ArcGIS 9.3 (ESRI, 2011).

Para los análisis estadísticos se comparó las unidades muestrales a través de la prueba de Kruskal-Wallis $(\alpha=0.05)$ en términos de acumulación de biomasa aérea (AGB est), bajo la presunción de que las muestras pudieran no ser similares (Ha). La prueba se realizó mediante el programa Past 2.17c (Hammer, Harper, \& Ryan, 2001). Posteriormente, el análisis de Escalamiento Multidimensional No
Paramétrico (NMDS) se realizó según la similitud de Bray-Curtis a partir de la función Log $\mathrm{x}+1$ para estandarizar los datos, desarrollado con el programa Primer-E 6.0 (Clarke, \& Gorley, 2006). Todos los datos ocupados en este estudio fueron previamente procesados y luego analizados con Microsoft ${ }^{\circledR}$ Excel 2003/XLSTAT@ (Addinsoft, Inc., 2012).

\section{RESULTADOS}

Durante la evaluación de las 38 PPM se registró un total de 22938 individuos y 1708 especies. En términos de biomasa aérea, en cada unidad muestral se obtuvo en promedio $209.88 \pm 31.79 \mathrm{Mg} / \mathrm{ha}$, que abarcan rangos desde $136.40 \mathrm{Mg} / \mathrm{ha}$ (PNY-07) hasta $286.03 \mathrm{Mg} / \mathrm{ha}$ (VG-COU-2), ver cuadro 1; estos son los valores totales incluyendo a la familia Myristicaceae R. Br. 


\section{艺}

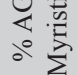

范

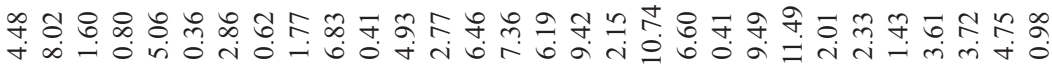

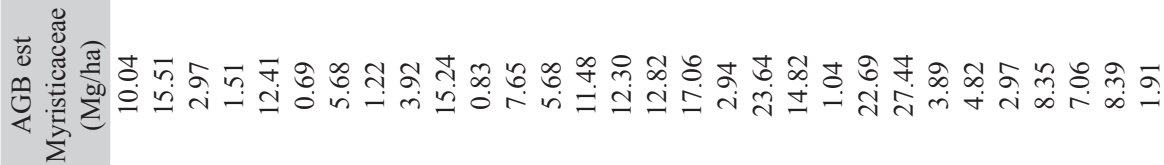

픙

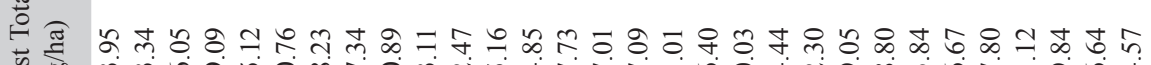

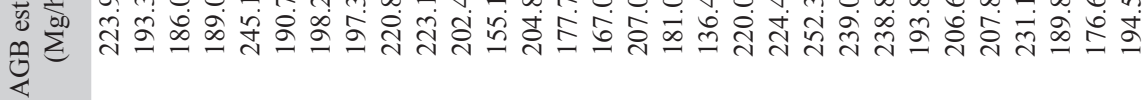

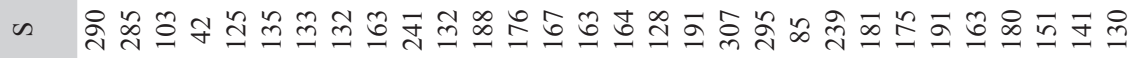

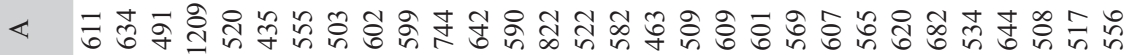

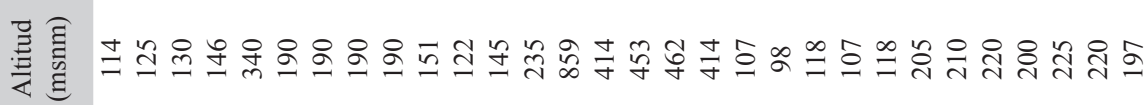

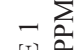

至衣

象党

竞

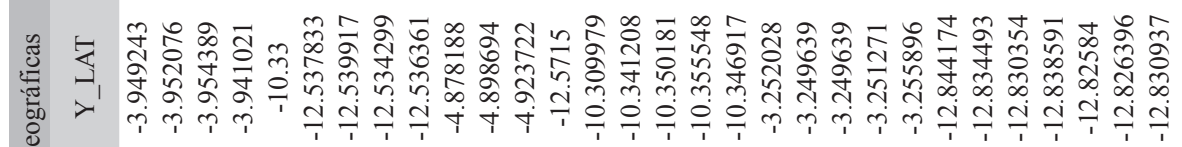

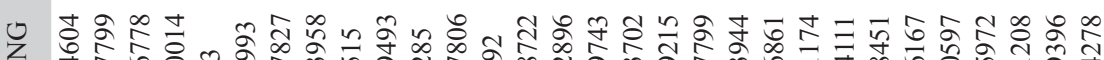

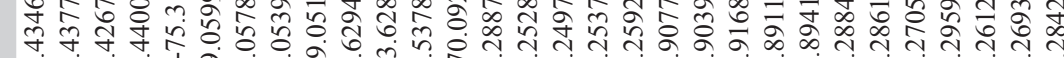

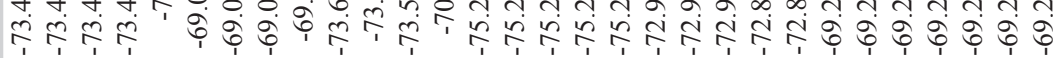

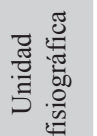

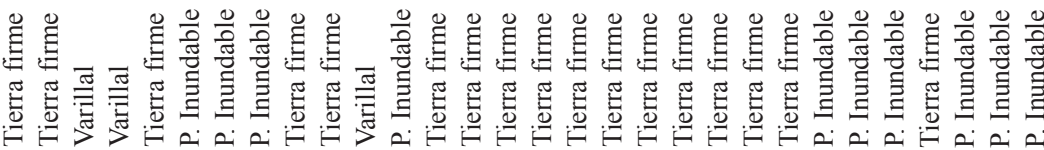

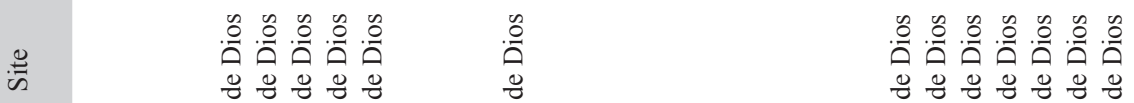

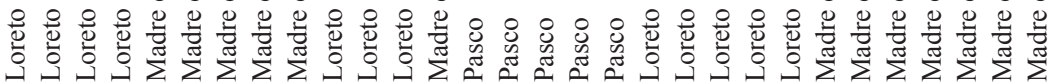

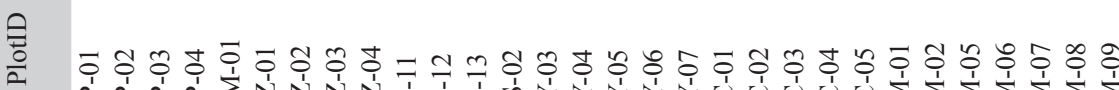

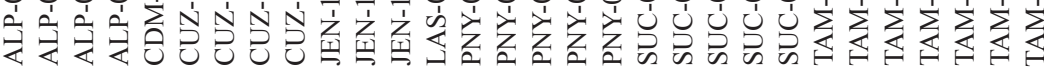
@ 


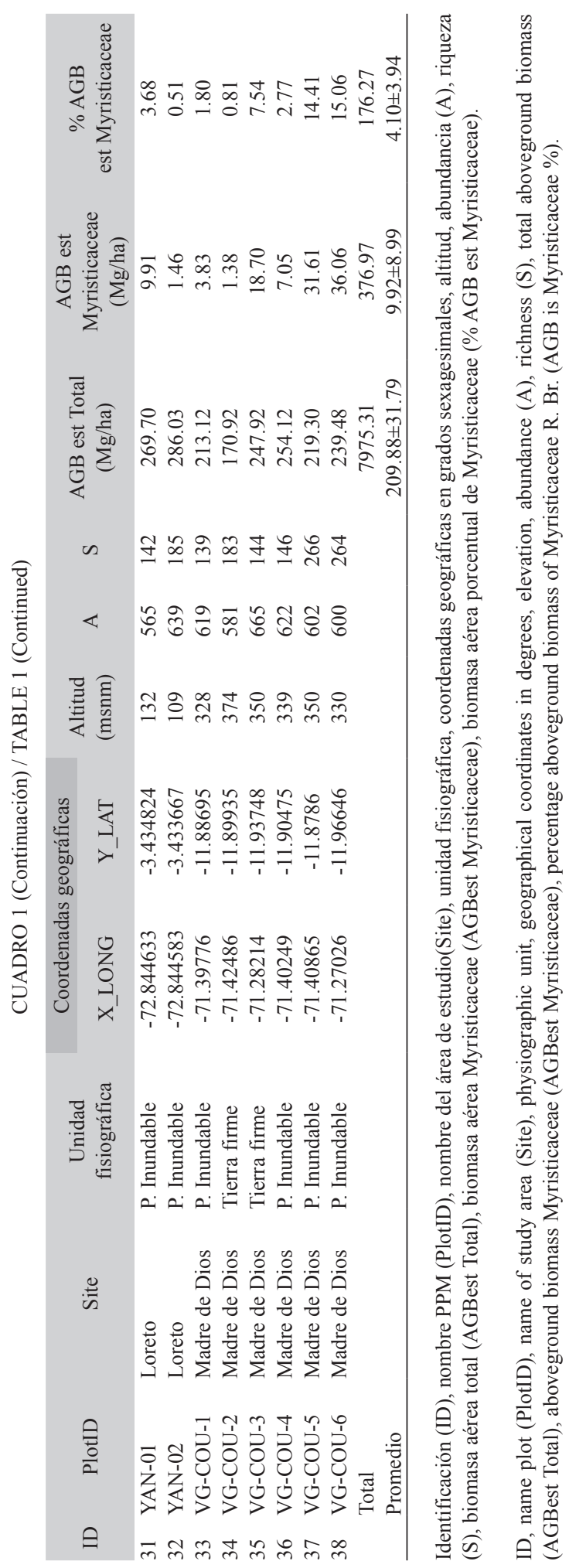




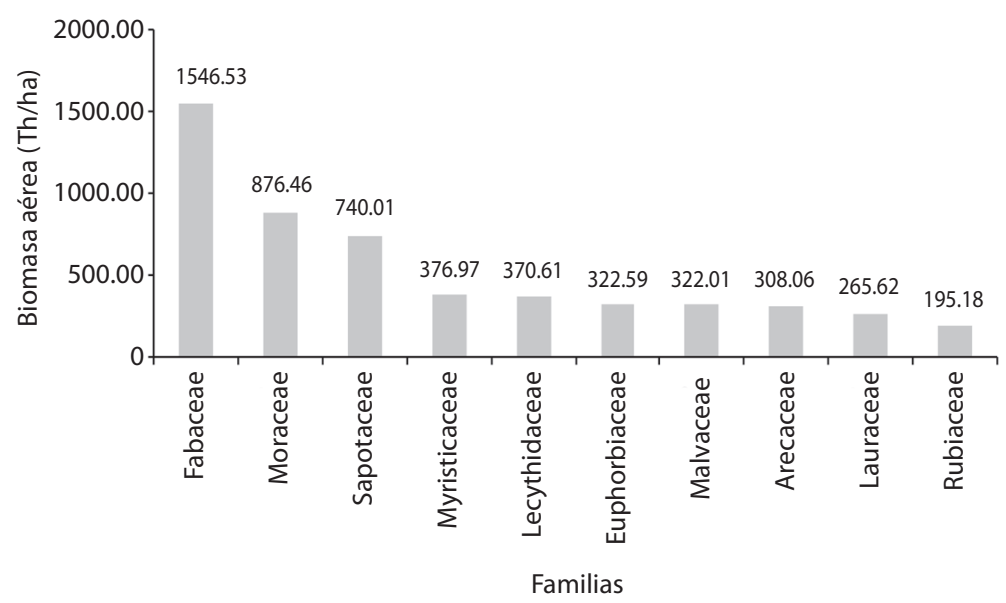

Fig. 2. Biomasa aérea acumulada de las 10 familias más representativas en las 38 PPM.

Fig. 2. Aboveground biomass of the 10 families more representative in 38 PPM.

En la figura 2 se muestran las 10 familias más importantes según su aporte de biomasa aérea acumulada, en el cual la familia interés, Myristicaceae R. Br., ocupó el cuarto lugar con $376.97 \mathrm{Mg} / \mathrm{ha}(9.92 \mathrm{Mg} / \mathrm{ha}$ en promedio) que representa el $4.73 \%$ del aporte sobre 91 familias evaluadas, registrado en 1702 individuos y 39 especies de Myristicaceae R. Br. (Cuadro 2). Entre las 39 especies de Myristicaceae R. Br., las especies que aportaron sumas considerables de biomasa aérea acumulada fueron Virola pavonis $(60.99 \mathrm{Mg} / \mathrm{ha})$, Otoba parvifolia $(52.74 \mathrm{Mg} / \mathrm{ha})$ y Osteophloeum platyspermum $(48.49 \mathrm{Mg} / \mathrm{ha})$, ellas se encuentran dentro de las 25 especies más importantes con este atributo registradas en las 38 PPM.

El análisis de superficie demostró que la mayor distribución de Myristicaceae R. Br. según la biomasa aérea acumulada está sobre la parte nordeste de la Amazonía (Fig. 1), agrupando cinco PPM con la mayor abundancia y riqueza de especies (Cuadro 1), y en un grado moderado pero homogéneo de distribución en la Amazonía sur.

La prueba de Kruskal-Wallis demostró que no existen diferencias significativas ( $p$-value $=0.1061)$ entre las 38 unidades muestrales (PPM) en términos de acumulación de biomasa aérea; pero si hay diferencias significativas $\left(p\right.$-value $\left.=4.603 \mathrm{E}^{-07}\right)$ entre muestras únicamente con especies de Myristicaceae R. Br., aceptando la hipótesis alternativa.

El análisis de ordenamiento (NMDS) para la familia Myristicaceae R. Br. resultó en una solución de dos dimensiones con un mínimo de estrés moderadamente bajo (estrés $=0.17$ ) dentro del ajuste Kruskal (Fig. 3). El análisis muestra tres agrupamientos bien definidos y con afinidad a ciertas unidades fisiográficas, lo que deja sueltas a las parcelas SUC-03 (menor aporte de AGBest $=0.69 \mathrm{Mg} / \mathrm{ha}$ ) y CUZ-01 (baja riqueza $=3$ ) ver cuadro 1 . El primer grupo y más grande (línea punteada, grupo a) corresponde a las PPM con mayor acumulación de biomasa aérea con una riqueza significativa y agrupa a especies que generalmente prefieren bosques de tierra firme como principal unidad fisiográfica, y excepcionalmente a dos PPM (YAN-01 y YAN-02) que comparten parte de su superficie con bosques de planicie inundable ubicado en las riberas de lo ríos con aguas blancas, ricos en sedimentos minerales o también denominado "várzea" como Virola elongata y Virola surinamensis. El segundo grupo (línea ploma, grupo b) que agrupa los valores más bajos en los tres parámetros mencionados (biomasa aérea acumulada, A y S) pero no menos importantes, ya que reúne a especies de Myristicaceae R. Br. con amplia preferencia sobre bosques de suelo arenoso, principalmente de 
CUADRO 2

Aporte de biomasa aérea por especie de Myristicaceae R. Br. Biomasa aérea acumulada en 38 PPM (AGB est acum.) y biomasa aérea promedio por PPM (AGB est prom.)

TABLE 2

Input from aboveground biomass by species of Myristicaceae R. Br. Aboveground biomass accumulated in 38 PPM (AGB is accumulated) and average aboveground biomass in PPM biomass (AGB is avg.)

\begin{tabular}{|c|c|c|}
\hline Species & $\begin{array}{c}\text { AGBest acum. } \\
\mathrm{Mg} / \mathrm{ha}\end{array}$ & $\begin{array}{c}\text { AGBest prom. } \\
\mathrm{Mg} / \mathrm{ha}\end{array}$ \\
\hline Compsoneura capitellata & 1.40 & 0.037 \\
\hline Compsoneura sprucei & 1.06 & 0.028 \\
\hline Iryanthera crassifolia & 1.21 & 0.032 \\
\hline Iryanthera elliptica & 3.97 & 0.104 \\
\hline Iryanthera juruensis & 27.28 & 0.718 \\
\hline Iryanthera laevis & 26.26 & 0.691 \\
\hline Iryanthera lancifolia & 2.97 & 0.078 \\
\hline Iryanthera macrophylla & 1.48 & 0.039 \\
\hline Iryanthera olacoides & 1.65 & 0.043 \\
\hline Iryanthera paradoxa & 2.15 & 0.057 \\
\hline Iryanthera paraensis & 7.40 & 0.195 \\
\hline Iryanthera polyneura & 1.12 & 0.029 \\
\hline Iryanthera sp. & 0.81 & 0.021 \\
\hline Iryanthera tessmannii & 0.24 & 0.006 \\
\hline Iryanthera tricornis & 11.07 & 0.291 \\
\hline Iryanthera ulei & 2.55 & 0.067 \\
\hline Osteophloeum platyspermum & 48.49 & 1.276 \\
\hline Otoba glycycarpa & 27.89 & 0.734 \\
\hline Otoba indet & 0.03 & 0.001 \\
\hline Otoba parvifolia & 52.74 & 1.388 \\
\hline Virola albidiflora & 3.46 & 0.091 \\
\hline Virola caducifolia & 0.14 & 0.004 \\
\hline Virola calophylla & 23.69 & 0.623 \\
\hline Virola decorticans & 0.51 & 0.014 \\
\hline Virola duckei & 15.13 & 0.398 \\
\hline Virola elliptica & 0.16 & 0.004 \\
\hline Virola elongata & 8.54 & 0.225 \\
\hline Virola flexuosa & 10.89 & 0.287 \\
\hline Virola loretensis & 0.99 & 0.026 \\
\hline Virola marlenei & 0.25 & 0.006 \\
\hline Virola minutiflora & 0.62 & 0.016 \\
\hline Virola mollissima & 0.46 & 0.012 \\
\hline Virola multiflora & 1.17 & 0.031 \\
\hline Virola multinervia & 2.44 & 0.064 \\
\hline Virola pavonis & 60.99 & 1.605 \\
\hline Virola peruviana & 0.81 & 0.021 \\
\hline Virola sebifera & 13.41 & 0.353 \\
\hline Virola sp. & 2.13 & 0.056 \\
\hline Virola surinamensis & 9.41 & 0.248 \\
\hline Total & 376.97 & 9.92 \\
\hline
\end{tabular}

arena blanca o también denominados "varillales" como Virola pavonis en ALP-30, ALP-40 y JEN-12, ubicados únicamente en Loreto. El último grupo (línea negra, grupo c) se agrupan PPM que presentan valores significativos de biomasa aérea y abundancia, pero con una deficiencia en la riqueza de especies de esta familia, las 17 especies en este grupo se distribuyen tanto en bosques de tierra firme como en bosques de planicie inundable pertenecientes a áreas inundadas por aguas negras y/o mixtas, podrían ser calificados como pantanales por ser ricos en materia orgánica, también denominados "igapó" como Virola flexuosa, Iryanthera tessmannii y Virola loretensis (Fig. 3).

\section{DISCUSIÓN}

La presencia de tres grupos definidos en la distribución espacial de Myristicaceae R. Br. se debe probablemente a la preferencia de hábitats o a ciertas unidades fisiográficas, principalmente a los bosques de tierra firme, por otro lado como lo mencionan Vásquez (1997, Vásquez et al., 2010); Reynel, Pennington, Pennington, Flores, \& Daza (2003); Pennington et al. (2004), Gonçalves, \& Gomes (2007) son en los bosques sobre suelo arenoso específicamente con arena blanca o varillal y a bosques de planicie inundable, con áreas inundadas tanto en aguas blancas o "várzea" y en aguas negras y/o mixtas o "igapó", en los cuales se observa una clara especificidad de algunas especies de esta familia con afinidades, adaptaciones y ciertas modificaciones morfológicas, demostrando que la heterogeneidad de cada área de muestreo puede influir en la distribución de un determinado grupo vegetal, lo que concuerda con Gentry, (1993); Vásquez (1995); Vásquez et al. (2005); Vásquez \& Rojas (2006); UlloaUlloa et al. (2004).

Geográficamente, es en el nororiente del Perú el lugar que albergó a la mayor riqueza y abundancia de especies de esta familia, que en cualquier otra área amazónica en el país como lo menciona Vásquez (1997). De forma similar se comportó el almacenamiento de biomasa aérea de la familia Myristicaceae, demostrando 


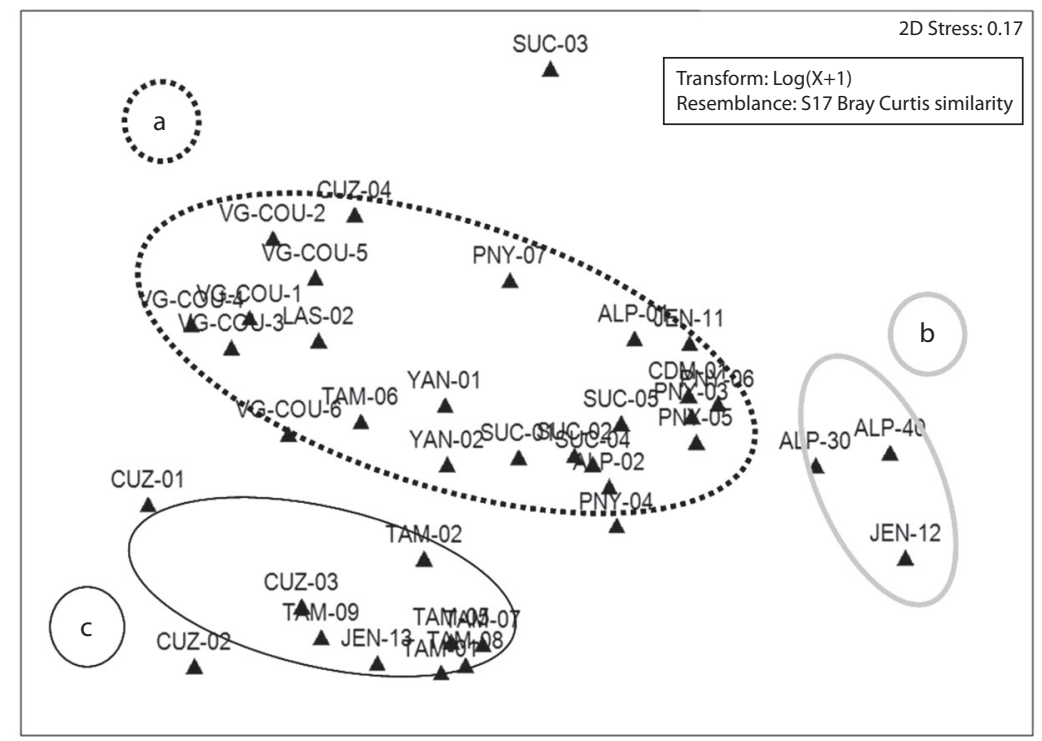

Fig. 3. Análisis de Escalamiento multidimensional no paramétrico (NMDS), clasificado según el valor acumulativo de biomasa aérea en las 38 PPM de la familia Myristicaceae R. Br, indicando el nivel de estrés en segunda dimensión. a) Grupo 1. b) Grupo 2. c) Grupo 3.

Fig. 3. Analysis of non-parametric multidimensional scaling (NMDS), classified according to the cumulative value of aboveground biomass in the 38 PPM of Myristicaceae R. Br. family indicating the level of stress in second dimension. a) Group 1. b) Group 2. c) Group 3.

que los valores más elevados de este atributo están en los bosques amazónicos, tal y como lo atribuye Overman, Witte, \& Saldarriaga (1994); Arreaga (2002); Baker et al. (2004 a, 2004b); Chave, Riéra, \& Dubois (2001); Chave et al., 2005; 2006a, 2006b); Mostacedo et al. (2006); Phillips et al. (1998; 2002; 2004; 2009); Malhi et al. (2009). Así mismo, el valor de biomasa aérea obtenido de 22938 árboles en todo el estudio, supera fácilmente a los valores encontrados en Brasil y Guyana Francesa, con rangos extremos para bosques húmedos tropicales que oscilan entre 28 a 423t/ ha, como menciona Chave et al. (2001), y el valor obtenido en este estudio se encuentra dentro del promedio de bosques amazónicos establecidos por RAINFOR que oscilan entrelos 216.96 a $299.20 \mathrm{Mg} /$ ha en un gradiente de 100 a $200 \mathrm{msnm}$ aproximadamente, obtenidos por Baker et al. (2004a), y que a pesar del valor promedio de biomasa aérea acumulada en la familia, son un componente esencial en términos cuantitativos al evaluar la composición florística amazónica, atribuyendo esto a su amplia diseminación por varios primates (Pithecia spp., Lagothrix spp., Callicebus spp.) y por algunas especies de tucanes (Ramphastos spp.), (van Roosmalen, 2003).

Finalmente, con este estudio se reafirma la idea de que ciertas especies de Myristicaceae R. Br., presentan una preferencia a determinadas unidades fisiográficas pero aún es necesario realizar estudios más profundos que mencionen los factores principales que inducen a que se presente este comportamiento en particular, ya que la mayoría de información disponible sólo menciona este rasgo de la familia por observación directa, restándole, de cierta forma, la representatividad que deberían tener las Myristicaceae R. Br. por su amplia distribución, dominante en términos de densidad de especies, abundante y como variable para evaluar la dinámica de los bosques amazónicos del Perú. 


\section{AGRADECIMIENTOS}

Esta investigación forma parte del proyecto "Catálogo de especies y distribución de Myristicaceae R. Br. en las Áreas Protegidas de la Selva Central", patrocinado por The Elizabeth E. Bascom Fellowships for Latin American Female Botanists MBG, y se realizó dentro del Programa del Máster Oficial de Biodiversidad en Áreas Tropicales y su Conservación de la Universidad Internacional Menéndez Pelayo-España, ambos patrocinados por el Missouri Botanical Garden. Agradezco a los administradores de las organizaciones RAINFOR y TeamNetwork por facilitarme el acceso a su base de datos para el desarrollo de este estudio. A Francisco Cabrero Sañudo por todo su apoyo en el planteamiento y la elaboración de esta investigación. A Rodolfo Vásquez Martínez por el apoyo institucional de parte del Jardín Botánico de Missouri-Programa Perú y el gran aporte en mi formación en botánica tropical. Un agradecimiento especial a Nuria Otmani de la Universidad Complutense de Madrid (España) y a Lucio Gil de la Universidad Internacional de Andalucía (España), por la corrección y revisión del manuscrito.

\section{RESUMEN}

Los bosques amazónicos son un gran almacén de biodiversidad y funcionan como sumideros de carbono debido a la biomasa aérea que acumulan en las diversas especies arbóreas, sin embargo, no se puede definir con exactitud cuál es el taxón con el mayor aporte de biomasa aérea. La representativa distribución de las Myristicaceae R. Br. en la Amazonía peruana fue el punto de inicio para plantear el presente estudio, el cual pretende cuantificar el aporte de biomasa aérea de esta familia en estos bosques. Para este estudio se utilizaron las bases de datos de 38 unidades muestrales recolectadas y proporcionadas por las organizaciones RAINFOR y TeamNetwork. Con estos datos se realizó la estimación de la biomasa aérea por medio de ecuaciones alométricas pre-establecidas, contraste de muestras con la prueba de Kruskal-Wallis, mapas de interpolación y un análisis de ordenamiento de escalamiento multidimensional no paramétrico (NMDS). Los resultados sugieren que Myristicaceae R. Br. es la cuarta familia más importante en aporte de biomasa aérea acumulada con $376.97 \mathrm{Mg} / \mathrm{ha}(9.92 \mathrm{Mg} / \mathrm{ha}$ en promedio) debido principalmente a su abundancia. Adicionalmente, la familia muestra una evidente preferencia de hábitat por determinadas condiciones edáficas dentro las unidades fisiográficas, tal es el caso de Virola pavonis en los "varillales", dentro de "planicies inundables", o de Iryanthera tessmannii y Virola loretensis en áreas inundadas de aguas negras o "igapó" específicamente, y de Virola elongata y Virola surinamensis en áreas inundadas de aguas blancas o "várzea".

Palabras clave: biomasa aérea, Amazonía peruana, Myristicaceae, preferencias, unidades fisiográficas.

\section{REFERENCIAS}

Arreaga, W. (2002). Almacenamiento del carbono en bosques con manejo forestal sostenible en la Reserva de Biosfera Maya, Petén, Guatemala (Tesis de Postgrado). Centro Agronómico Tropical de Investigación y Enseñanza, Costa Rica.

Baker, T., Phillips, O., Malhi, Y., Almeida, S., Arroyo, L., Di Fiore, A., \& Vásquez, R. (2004a). Increasing biomass in Amazonian forest plots. Philosophical Transactions of the Royal Society of London B: Biological Sciences, B359, 353-365.

Baker, T., Phillips, O., Malhi, Y., Almeida, S., Arroyo, L., Di Fiore, A., \& Vásquez, R. (2004b). Variation in wood density determines spatial patterns in Amazonian forest biomass. Global Change Biology, 10, $545-562$.

Baker, T., Phillips, O., Laurance, W., Pitman, N., Almeida, S., Arroyo, L., \& Lloyd, J. (2009). Do species traits determine patterns of wood production in Amazonian forests? Biogeosciences, 6, 297-307.

Brako, L., \& Zarucchi, J. L. (1993). Catálogo de las Angiospermas y Gimnospermas del Perú. Monographs in Systematic Botany from the Missouri Botanical Garden, 45, 428-429.

Chave, J., Riéra, B., \& Dubois, M. (2001). Estimation of Biomass in a Neotropical Forest of French Guiana: Spatial and Temporal Variability. Journal of Tropical Ecology, 17(1), 79-96.

Chave, J., Andalo, C., Brown, S., Cairns, M., Chambers, J., Eamus, D., \& Yamakura, T. (2005). Tree allometry and improved estimation of carbon stocks and balance in tropical forests. Ecosystem Ecology Oecologia, 145, 87-99.

Chave, J., Muller Landau, H., Baker, T., Easdale, T., ter Steege, H., \& Webb, C. (2006a). Regional and phylogenetic variation of wood density across 2,456 neotropical tree species. Ecological Applications, 16, 2356-2367.

Chave, J. (2006b). Medición de densidad de madera en árboles tropicales-Manual de campo. France: Sixth Framework Programme Pan-Amazonia.

Clarke, K., \& Gorley, R. (2006). PRIMER v6: User Manual/Tutorial. Plymouth, UK: PRIMER-E. 
Cornejo, F., Janovec, J., \& Tobler, M. (2006). Floristic diversity and composition of terra firme and seasonally inundated palm swamp forests in the palma real watershed in lower Madre De Dios, Peru. Sida, 22(1), 615-633.

Environmental Systems Research Institute (ESRI). (2011). ArcGIS Desktop: Release 10. Redlands, CA.

Freitas, S., Longo, K., Silva-Dias, M., Silva-Dias, P., Chatfield, R., Prins, E., \& Recuero, F. (2005). Monitoring the transport of biomass burning emissions in South America. Environmental Fluid Mechanics, 5, 135-167.

Gentry, A. (1982). Neotropical floristic diversity: Phytogeographical connections between Central and south America, Pleistocene climatic fluctuations, or an accident of the Andean orogeny? Annales Missouri Botanical Garden, 69, 557-593.

Gentry, A. (1986). Endemism in tropical versus temperature plant communities. In M. Soulé (Ed.), Conservation Biology (pp. 153-181). Sunderland, MA: Sinauer Press.

Gentry, A. (1993). A Field Guide to the Families and Genera of Woody Plants of Northwest South America (Colombia, Ecuador, Perú) with supplementary notes on herbaceous taxa. Washington: Conservation International.

Gentry, A., \& Ortiz, R. (1993). Patrones de composición florística en la Amazonía Peruana. In R. Kalliola, M. Puhakka, \& W. Danjoy (Eds.), Amazonía Peruana: Vegetación húmeda tropical en el llano subandino (pp. 155-166). PAUT-ONERN. Jyväskylä, Finland: Gummerus Printing.

Gonçalves, M., \& Gomes, C. (2007). Biologia floral de Virola surinamensis (Rol.) Warb. (Myristicaceae). Revista. Árvore, 31(6), 1155-1162.

Hammer, Ø., Harper, D., \& Ryan, P. (2001). PAST: Paleontological statistics software package for education and data analysis. Palaeontologia Electronica, $4(1), 9$.

Holdridge, L. (1947). Determination of world plant formation from simple climatic data. Science, 105, 367-368

Javonec, J. (2002). Compsoneura diazii (Myristicaceae R. Br.), A new species from the río Cenepa area of northwestern Peru. Missouri Botanical Garden, 12, 366-368.

Keller, M., Alencar, A., Asner, G., Braswell, B., Bustamente, M., Davidson, E., \& Vourlitis, G. (2004). Ecological research in the Large-scale BiosphereAtmosphere experiment in Amazonia: Early results. Ecological Applications, 14, 3-16.

Magnusson, W., Costa, F., Lima, A., Baccaro, F., BragaNeto, R., Laerte Romero, R., \& Lawson, B. (2008).
A program for monitoring biological diversity in the Amazon: An alternative perspective to threat-based monitoring. Biotropica, 40, 409-411.

Malhi, Y., Wood, D., Baker, T., Wright, J., Phillips, O. L., Cochrane, T., \& Vinceti, B. (2006). The Regional variation of above-ground live biomass in old-growth Amazonian forests. Global Change Biology, 12, 1107-1138.

Malhi, Y., Aragão, L., Metcalfe, D., Paiva, R., Quesada, C., Almeida, S., \& Teixeira, L. (2009). Comprehensive assessment of carbon productivity, allocation and storage in three Amazonian forests. Global Change Biology, 1-20.

Mostacedo, B., Villegas, Z., Peña, M., Pooter, L., Licona, J., \& Alarcon, A. (2006). Fijación de carbono (biomasa aérea) en áreas de manejo forestal sujetas a diferentes intensidades de aprovechamiento: Implicaciones a corto y mediano plazo. Bolivia: Instituto boliviano de investigación forestal (IBIF)-Embajada de Holanda.

Overman, J., Witte, H., \& Saldarriaga, J. (1994). Evaluation of Regression Models for Above-Ground Biomass Determination in Amazon Rainforest. Journal of Tropical Ecology, 10(2), 207-218.

Pascal, J., \& Pelissier, S. (1996). Structure and floristic composition of a tropical evergreen forest in southwest India. Journal of Tropical Ecology, 12, 191-214.

Pennington, D., Reynel, C., \& Daza, A. (2004). Illustrated guide to the Trees of Peru. England: SpoelbeechArtois Foundation.

Phillips, O., Malhi, Y., Higuchi, N., Laurance, W., Nuñez, P., Vásquez, R., \& Grace, J. (1998). Changes in the carbon balance of tropical forests: evidence from long-term plots. Science, 282(16), 439-442.

Phillips, O., Malhi, Y., Vinceti, B., Baker, T., Lewis, S. L., Higuchi, N., \& Grace, J. (2002). Changes in growth of tropical forests: Evaluating potential biases. Ecological Applications, 12(2), 576-587.

Phillips, O., \& Baker, T. (2003). Manual de campo para el establecimiento y remedición de parcelas permanentes. Cantua, 12, 85-93.

Phillips, O., Baker, T., Arroyo, L., Higuchi, N., Killeen, T., Laurance, W., \& Vinceti, B. (2004). Pattern and process in Amazon tree turnover, 1976-2001. Philosophical Transactions of the Royal Society of London Biological Sciences, 359, 381-407.

Phillips, O., Aragão, L., Lewis, S., Fisher, J., Lloyd, J., López-González, G., \& Torres-Lezama, C. (2009). Drought sensitivity of the Amazon rainforest. Science, 323, 1344-1347.

Prentice, I., Farquhar, G., Fasham, M., Goulden, M., Heimann, M., Jaramillo, V., \& Wallace, D. (2001). The 
carbon cycle and atmospheric carbon dioxide. In J. Houghton, \& D. Yihui (Eds.), Climate Change: The Scientific Basis (pp. 183-197). Cambridge: Cambridge University Press.

Poulsen, A., Nelson, I., Tan, S., \& Balsleb, H. (1996). A quantitative inventor of trees in one hectare of mixed dipterocarp forest in Temburong. In D. S. Edwards, W. E. Booth, \& S. C. Choy (Eds.), Brunei Darussalarn (pp. 139-150). London, England: Tropical Rainforest Research-Current Issues, Kluwer Academic Publishers.

Reynel, C., Pennington, D., Pennington, T., Flores, C., \& Daza, A. (2003). Árboles útiles de la Amazonía Peruana. Perú: Darwin Initiative Project 09/017, International Center for Research in Agroforestry-ICRAF.

Ribeiro, J., Hopkins, M., Vicentini, A., Sothers, C., Costa, M., Brito, J., \& Procópio, L. (1999). Flora da Reserva Ducke - Guia de identificação das plantas vasculares de uma floresta de terra-firme na Amazônia Central. Manaus, Brasil: INPA/DFID.

Saatchi, S., Houghton, R., Dos Santos, A., Soares, J., \& Yu, Y. (2007). Distribution of aboveground live biomass in the Amazon basin. Global Change Biology, 13, 816-837.

Spichiger, R.,. Loizeau, P., Latou, C., \& Barriera, G. (1996). Trees species richness in a southwestern Amazonian forest. Candollea, 51, 559-577.
Ulloa-Ulloa, C., Zarucchi, J., \& León, J. (2004). Diez años de adiciones a la flora del Perú: 1993-2003. Arnaldoa, 1-242.

van Roosmalen, M. (2003). Myristicaceae. Amazonas, Brazil: Instituto Nacional de Pesquisas da Amazônia (INPA).

Vásquez, R. (1995). Árboles de la amazonía nororiental del Perú: Diversidad, destrucción y conservación. Missouri Botanical Garden. Arnadoa, 3(2), 73-86.

Vásquez, R. (1997). Flórula de las Reservas Biológicas de Iquitos, Perú. St. Louis, USA: Missouri Botanical Garden.

Vásquez, R., Rojas, R., Monteagudo, A., \& Meza, K. (2005). Flora Vascular de la selva central del Perú: Una aproximación de la composición florística de tres Áreas Naturales Protegidas. Missouri Botanical Garden. Arnaldoa, 12(1-2), 112-125.

Vásquez, R., \& Rojas, R. (2006). Plantas de la Amazonía Peruana. Missouri Botanical Garden. Arnaldoa, 13(1), 1-258.

Vásquez, R., Rojas, R., \&Van der Werff, H. (2010). Flora del Río Cenepa, Amazonas, Perú. USA: Missouri Botanical Garden.

Vieira, S., Ferreira, L., Aidar, M., Spinelli, L., Baker, T., Ferreira, J., \& Trumbore, S. (2008). Estimation of biomass and carbon stocks: the case of the Atlantic Forest. Biota Neotropical, 8(2), 21-29. 
